\title{
Intrinsic errors of the central galactic mass derived from rotation curves under the influence of a weak non-axisymmetric potential
}

\author{
J. Koda ${ }^{1,2, \star}$ and K. Wada ${ }^{2}$ \\ 1 Institute of Astronomy, University of Tokyo 2-21-1, Osawa, Mitaka, Tokyo 181-0015, Japan \\ 2 National Astronomical Observatory, 2-21-1, Osawa, Mitaka, Tokyo 181-8588, Japan \\ Received 12 July 2002 / Accepted 1 October 2002
}

\begin{abstract}
Rotation curves are often used to estimate the mass distribution of spiral galaxies, assuming that the circular velocities of the interstellar medium balance with the galactic centrifugal force. However, non-circular motions caused by a non-axisymmetric gravitational potential, such as a stellar bar, may disturb the velocity field, resulting in errors in mass estimation, especially in the central regions of galaxies. This is because the line-of-sight velocity depends on the viewing angles in a non-axisymmetric flow. Observing rotation curves of edge-on galaxies in time-dependent numerical simulations from different viewing angles, we obtain errors in the estimation of galactic mass from the rotation curves. In the most extreme case, the ellipticity of gas orbits is as high as $e \sim 0.8$ in the central regions, even if the bar potential is weak. When rotation curves are defined as the highest velocity envelope of position-velocity diagrams, the mass estimated from the rotation curves is larger than the true mass by a factor of five for $15 \%$ of the viewing angles, and the ratio between the apparent mass and true mass is less than six for any viewing angle. The overestimation in mass occurs more frequently than the underestimation.
\end{abstract}

Key words. galaxies: fundamental parameters (masses) - galaxies: ISM - galaxies: kinematics and dynamics - hydrodynamics

\section{Introduction}

Rotation curves are a major tool for determining mass distribution in spiral galaxies. Assuming spherical mass distribution, the galactic mass $M$ within a galactocentric radius $R$ is estimated by

$M=R V^{2} / G$,

where $V$ and $G$ stand for a circular velocity and gravitational constant.

Rotation curves in their outer regions are generally flat (Rubin et al. 1980, 1982, 1985), indicating massive dark halos surrounding their optical disks (Kent 1987). In their central regions, many rotation curves rise steeply from the centers, reaching the high velocity seen in the outer flat rotation curves: typically $100-300 \mathrm{~km} \mathrm{~s}^{-1}$ within a central $100 \mathrm{pc}$ radius (Sofue 1996; Sofue et al. 1999). These high velocities may indicate central massive cores of about $10^{9} M_{\odot}$ within a central 100 pc radius (Sofue 1996; Sofue et al. 1999; Takamiya \& Sofue 2000).

However, the gas in galactic disks does not necessarily show pure circular rotation, especially in the central regions. Bar-like distortions of the stellar system can drive non-circular

Send offprint requests to: J. Koda,

e-mail: jin.koda@nao.ac.jp

* JSPS Research Fellow; Present address: ALMA-J Project Office, National Astronomical Observatory, 2-21-1, Osawa, Mitaka, Tokyo, 181-8588, Japan. (elliptical) motions for the gas. As a result, the apparent rotation curves do not represent the correct mass distribution. For example, if the elliptical orbits were aligned by chance with the line-of-sight, we would overestimate the mass (Sakamoto et al. 1999). The effect of non-circular motion on position-velocity diagrams has been intensively studied in theoretical calculations (Bureau \& Athanassoula 1999; Athanassoula \& Bureau 1999).

In this paper, we quantitatively study the errors in estimating the mass from rotation curves in galaxies with a weak bar, and calculate the probability that the observed mass suffers from such errors. Even if the bar-like distortion of the gravitational potential is very weak, the gas velocity-field can be non-axisymmetric (Wada 1994). In order to obtain the velocityfield of the gas in a weak bar potential, we performed Smoothed Particle Hydrodynamics (SPH) simulations (Sect. 2). Using the numerical results, we estimate the probability of overestimating the central galactic mass (Sect. 3). We discuss the implications of our results in Sect. 4.

\section{Numerical calculations}

\subsection{2-D SPH methods}

We perform two-dimensional SPH calculations (Lucy 1977; Gingold \& Monaghan 1977), and reproduce gas motions in a weak stellar bar potential. We adopt the SPH formulation by Benz (1990), and use the spline kernel (Monaghan \& Lattanzio 1985) with the modification for its gradient 
(Thomas \& Couchman 1992). The correction term for viscosity (Balsara 1995) is taken into account to avoid large entropy generation in pure shear flows. The SPH smoothing length $h$ varies in space and time, keeping the number of particles within the radius $2 h$ at an almost constant of 32 according to the method of Hernquist \& Katz (1989). The leapfrog integrator is adopted to update positions and velocities. We use $3 \times 10^{4}$ particles to represent the gas disk.

\subsection{Galaxy disk and weak bar potentials}

We take the galaxy potential for a weak bar used in Sanders (1977) and Wada \& Habe (1992); the oval potential for a barred galaxy $\Phi(R, \theta)$ is represented in axisymmetric and bisymmetric parts by

$\Phi(R, \theta)=\Phi_{0}(R)+\Phi_{b}(R) \cos 2 \theta$,

where the first term is an axisymmetric potential for a disk,

$\Phi_{0}(R)=-C \frac{1}{\left(R^{2}+a^{2}\right)^{1 / 2}}$,

and the second term is a bisymmetric one for a weak bar,

$\Phi_{b}=-\epsilon_{0} C \frac{a R^{2}}{\left(R^{2}+a^{2}\right)^{2}}$.

The normalization coefficient $C=(27 / 4)^{1 / 2} a v_{\max }^{2}$ is calculated from the core radius $a$ and maximum rotation velocity $v_{\max } \cdot \epsilon_{0}$ is a parameter to control the strength of the bar. $R$ and $\theta$ stand for the galactocentric radius and azimuthal angle from the bar respectively. We are interested in the notion that noncircular motions in a bar apparently indicate a central massive component. We thus do not consider any massive central component in our potential, such as a bulge (Athanassoula \& Bureau 1999) or a massive black hole (Fukuda et al. 1998, 2000).

Our potential model has the benefit of being capable of analytically investigating gaseous orbits in the bar potential (Wada 1994), and has been well-studied in numerical simulations for the bar-driven gas fueling into galactic centers (Wada \& Habe 1992, 1995), the gas kinematics in the Galaxy (Wada et al. 1994), the spatial distribution of mass-to-light ratio in a galaxy NGC 4321 (Wada et al. 1998), and the effects of a central black hole (Fukuda et al. 1998, 2000).

We fix $a=\sqrt{2} \mathrm{kpc}$ and $v_{\max }=220 \mathrm{~km} \mathrm{~s}^{-1}$; the corresponding rotation curve is shown in Fig. 1. The gas reaches the maximum circular rotation velocity at $R=2 \mathrm{kpc}$ with a rotational period of $56 \mathrm{Myr}$. Figure 2 shows the radial changes of frequencies, $\Omega$ and $\Omega \pm \kappa / 2$, where $\Omega$ and $\kappa$ are circular and epicyclic frequencies respectively. We set the pattern speed of the bar $\Omega_{\mathrm{b}}$ at $0.4,0.8$, and 1.5 times the maximum of $\Omega-\kappa / 2$, indicated by horizontal lines. Models with $\Omega_{\mathrm{b}}=0.4$ and $0.8 \times(\Omega-\kappa / 2)_{\max }$ have two inner Lindblad resonances (ILR), while those with $\Omega_{\mathrm{b}}=1.5 \times(\Omega-\kappa / 2)_{\max }$ have no ILR. $\epsilon_{0}$ is set to $0.05,0.10$, and 0.15 . Our nine models are listed in Table 1.

\subsection{Initial conditions}

The gas is initially distributed in a uniform-density disk with an $8 \mathrm{kpc}$ radius, following pure circular-rotation that balances

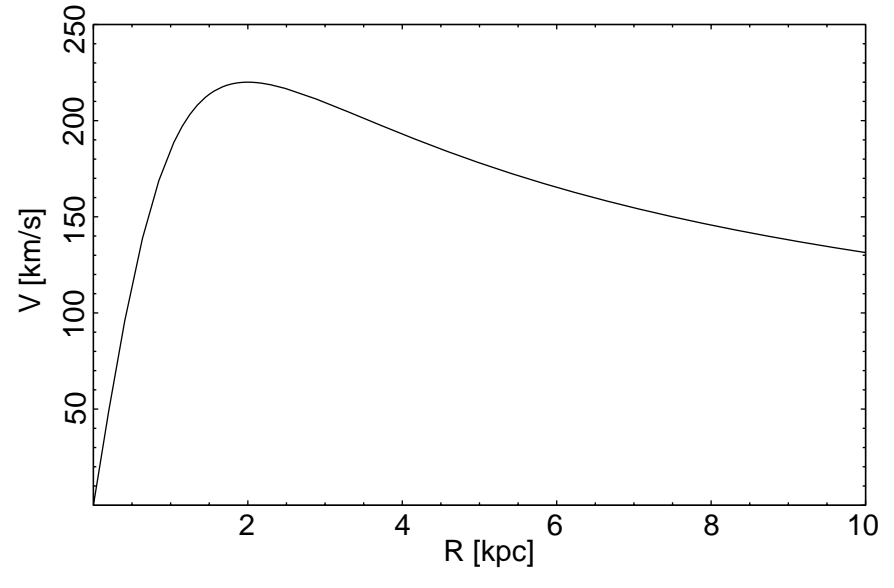

Fig. 1. Rotation curve from the axisymmetric potential, Eq. (3), with the core radius $a=\sqrt{2} \mathrm{kpc}$ and maximum rotation velocity $v_{\max }=$ $220 \mathrm{~km} \mathrm{~s}^{-1}$.

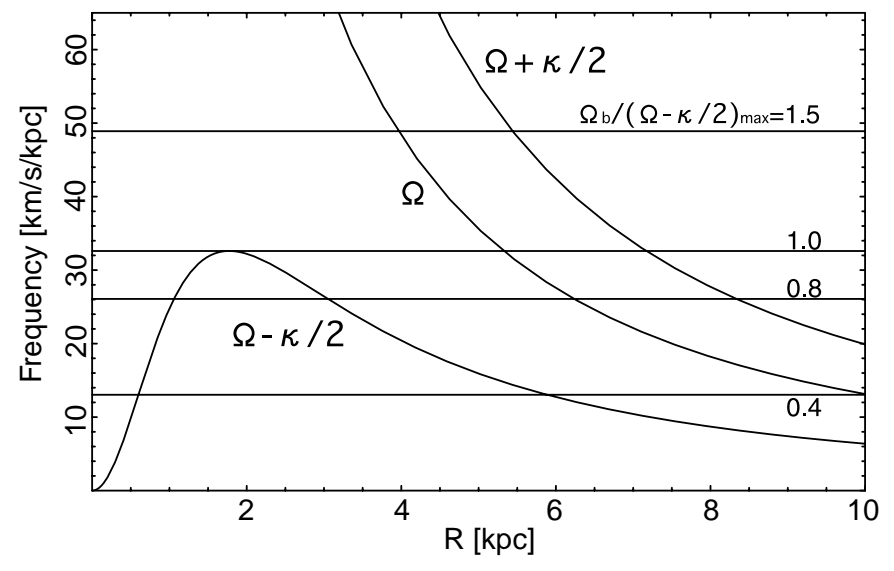

Fig. 2. Radial changes of frequencies, $\Omega(R)$ and $\Omega(R) \pm \kappa(R) / 2$. Four horizontal lines represent the pattern speeds of the bar, i.e. $0.4,0.8$, 1.0 , and $1.5 \times(\Omega-\kappa / 2)_{\max }$.

the centrifugal force. The gas temperature is assumed to be a constant $10^{4} \mathrm{~K}$, corresponding to the sound speed of about $10 \mathrm{~km} \mathrm{~s}^{-1}$, throughout evolution. The total gas mass is assumed to be $5 \%$ of the total stellar mass within the radius of $8 \mathrm{kpc}$. The results are not significantly affected by the total gas mass, because thermal pressure is much smaller than the rotational energy, and we do not calculate self-gravity of the gas. We advance the calculations up to about $500 \mathrm{Myr}$.

\subsection{Gas dynamical evolution}

Gas dynamics in a barred potential have been well studied in numerical simulations (Wada \& Habe 1992; Heller \& Shlosman 1994; Piner et al. 1995; Fukuda et al. 1998; Athanassoula \& Bureau 1999). Our models evolve consistently with these simulations. Figure 3, model E, shows a typical evolution. Three phases of the evolution can be seen in this model: (a) linear perturbation phase, $t \sim 0-50 \mathrm{Myr}$, (b) transient phase, $t \sim 50-250 \mathrm{Myr}$, and (c) quasi-steady phase, $t>250 \mathrm{Myr}$.

The characteristic structure appearing during the evolution depends strongly on the positions of resonances, i.e. the 
Table 1. Model parameters.

\begin{tabular}{|c|c|c|c|c|c|c|c|c|c|}
\hline \multirow[t]{2}{*}{ model } & \multicolumn{2}{|c|}{$\Omega_{\mathrm{b}}$} & \multirow[t]{2}{*}{$\epsilon_{0}$} & \multicolumn{2}{|c|}{ ILRs } & \multirow{2}{*}{$\begin{array}{c}\mathrm{CR} \\
(\mathrm{kpc})\end{array}$} & \multirow{2}{*}{$\begin{array}{l}\text { OLR } \\
(\mathrm{kpc})\end{array}$} & \multirow{2}{*}{$\begin{array}{c}\left(\rho_{\mathrm{b}} / \rho_{0}\right)_{\max } \\
(\%)\end{array}$} & \multirow{2}{*}{$\begin{array}{c}T_{\mathrm{bar}} \\
(\mathrm{Myr})\end{array}$} \\
\hline & $\left(\mathrm{km} \mathrm{s}^{-1} \mathrm{kpc}^{-1}\right)$ & $(\Omega-\kappa / 2)_{\max }$ & & $(\mathrm{kpc})$ & $\overline{(\mathrm{kpc})}$ & & & & \\
\hline$A \ldots \ldots$ & 49 & 1.5 & 0.05 & - & - & 4.0 & 5.4 & 5 & 130 \\
\hline$B \ldots \ldots$ & 49 & 1.5 & 0.10 & - & - & 4.0 & 5.4 & 10 & 130 \\
\hline C..... & 49 & 1.5 & 0.15 & - & - & 4.0 & 5.4 & 16 & 130 \\
\hline$D \ldots \ldots$ & 26 & 0.8 & 0.05 & 1.1 & 3.1 & 6.2 & 8.3 & 5 & 240 \\
\hline$E \ldots \ldots$ & 26 & 0.8 & 0.10 & 1.1 & 3.1 & 6.2 & 8.3 & 10 & 240 \\
\hline$F \ldots \ldots$ & 26 & 0.8 & 0.15 & 1.1 & 3.1 & 6.2 & 8.3 & 16 & 240 \\
\hline$G \ldots .$. & 13 & 0.4 & 0.05 & 0.6 & 5.9 & 10.0 & 13.3 & 5 & 470 \\
\hline$H \ldots \ldots$ & 13 & 0.4 & 0.10 & 0.6 & 5.9 & 10.0 & 13.3 & 10 & 470 \\
\hline$I \ldots \ldots$ & 13 & 0.4 & 0.15 & 0.6 & 5.9 & 10.0 & 13.3 & 16 & 470 \\
\hline
\end{tabular}

Two free parameters, i.e. bar pattern speed $\Omega_{\mathrm{b}}$ and bar strength $\epsilon_{0}$, and radii of inner Lindblad resonances (ILR), corotation resonance (CR) and outer Lindblad resonance (OLR), maximum density ratio of the bar over the disk $\left(\rho_{\mathrm{b}} / \rho_{0}\right)_{\max }$, and rotational time-scale of the bar $T_{\text {bar }}\left[\equiv 2 \pi / \Omega_{\mathrm{b}}\right]$ at $R=2 \mathrm{kpc}$ are tabulated.

pattern speed of the bar $\Omega_{\mathrm{b}}$. In phase (a), leading and trailing spiral arms are formed around the inner $(R=1.1 \mathrm{kpc})$ and outer $(3.1 \mathrm{kpc})$ ILRs respectively at $t=36 \mathrm{Myr}$. These resonant-driven spirals are expected in a linear theory (Wada 1994). While the outer trailing arms remain with increasing density contrasts, the inner leading arms evolve into an oval ring, or a gaseous bar $(t=71-107 \mathrm{Myr})$, i.e. phase (b). The oval ring first leads the stellar bar $(71 \mathrm{Myr})$, rotating opposite to the gas rotation $(107 \mathrm{Myr})$, and being aligned with the stellar bar (250 Myr), and thereafter, the system develops toward a quasi-steady phase, i.e. phase (c). The ellipticity of the nuclear ring grows as high as $e \sim 0.8$. The ripple seen in the outer arms at $t=394 \mathrm{Myr}$ would originate in the Kelvin-Helmholtz instability (Piner et al. 1995). Gas dynamics and structure in the inner region of the disk are not affected by this instability.

Figures 4 and 5 display the final snapshots and velocity fields for nine models. Different pattern speeds $\Omega_{\mathrm{b}}$ and bar strength $\epsilon_{0}$ are arranged vertically and horizontally, respectively. It is evident that the final structure depends strongly on $\Omega_{\mathrm{b}}$, while $\epsilon_{0}$ changes only the density contrasts. Model A, B, and $C$ have no ILRs, thus no spiral arms or ring in their inner regions are formed. The outer spiral arms are formed outside the radius of the corotation resonance $(\mathrm{CR})$ due to the outer Lindblad resonance (OLR). Model D and F resemble model E. Model G, $\mathrm{H}$ and I also have arms and rings similar to those in model $\mathrm{E}$, but at different radii, corresponding to the location of the ILRs. Figure 5 clearly show that, in models D, E, F and G, most gaseous orbits are $x_{1}$-like, while in model $\mathrm{H}$ and $\mathrm{I}$, the large separation and low density between the two ILRs suffice to leave the gases on $x_{2}$-orbits, which form a stable oval ring, nearly perpendicular to the stellar bar.

\section{Galactic mass derived from rotation curves}

In this section, we compare the apparent rotation curves obtained from the numerical results (Sect. 3.1) and the true rotation curves, then we get probability to overestimate/underestimate the galactic mass (Sects. 3.2 and 3.3).

\subsection{Observing rotation curves in models}

We obtain a position-velocity (p-v) diagram by observing our calculated gas disks edge-on, and then determine a rotation curve from the $\mathrm{p}-\mathrm{v}$ diagram. We assign gas particles in a position-velocity grid using the cloud-in-cell method (Hockney \& Eastwood 1981); the spacing for the grid is set to a typical resolution in recent interferometry observations of the $\mathrm{CO}$ gas for Virgo galaxies, i.e. $100 \mathrm{pc}\left(\sim 1^{\prime \prime}\right)$ in space and $5 \mathrm{~km} \mathrm{~s}^{-1}$ in velocity (Sofue in private communication). Then we determine a rotation curve by tracing the gas at the highest velocity for each radius in the $\mathrm{p}-\mathrm{v}$ diagram. Some examples for $\mathrm{p}-\mathrm{v}$ diagrams and rotation curves are shown in Fig. 6.

There are several ways to derive a rotation curve from an observed $p-v$ diagram. One traces the peak-intensity velocity or intensity-weighted mean velocity at each radius (Rubin et al. 1980, 1982, 1985; Mathewson et al. 1992; Mathewson \& Ford 1996), while another traces the $20 \%$ envelope of the peakintensity velocity at each radius (Sofue 1996). These intensitybased methods cannot be applied to our density-based p-v diagram, because intensity is not a simple function of density, especially in edge-on systems. Compared with these methods, our method provides generally higher velocity.

\subsection{Error estimation in rotation curves and mass}

We compare the observed rotation curves $V_{\text {obs }}(R)$ derived from p-v diagrams in simulations (Sect. 3.1), with the true rotation curves $V_{\text {pot }}(R)$ from the gravitational potential $\left(\Phi_{0}(R)\right)$. We estimate the errors in rotation curves by defining a function, i.e.

$\alpha(R) \equiv \frac{V_{\mathrm{obs}}(R)}{V_{\mathrm{pot}}(R)}$

and examining whether $\alpha$ exceeds an arbitrary critical value $\alpha_{\text {crit }}$ as

$\alpha(R) \geq \alpha_{\text {crit }}$ 

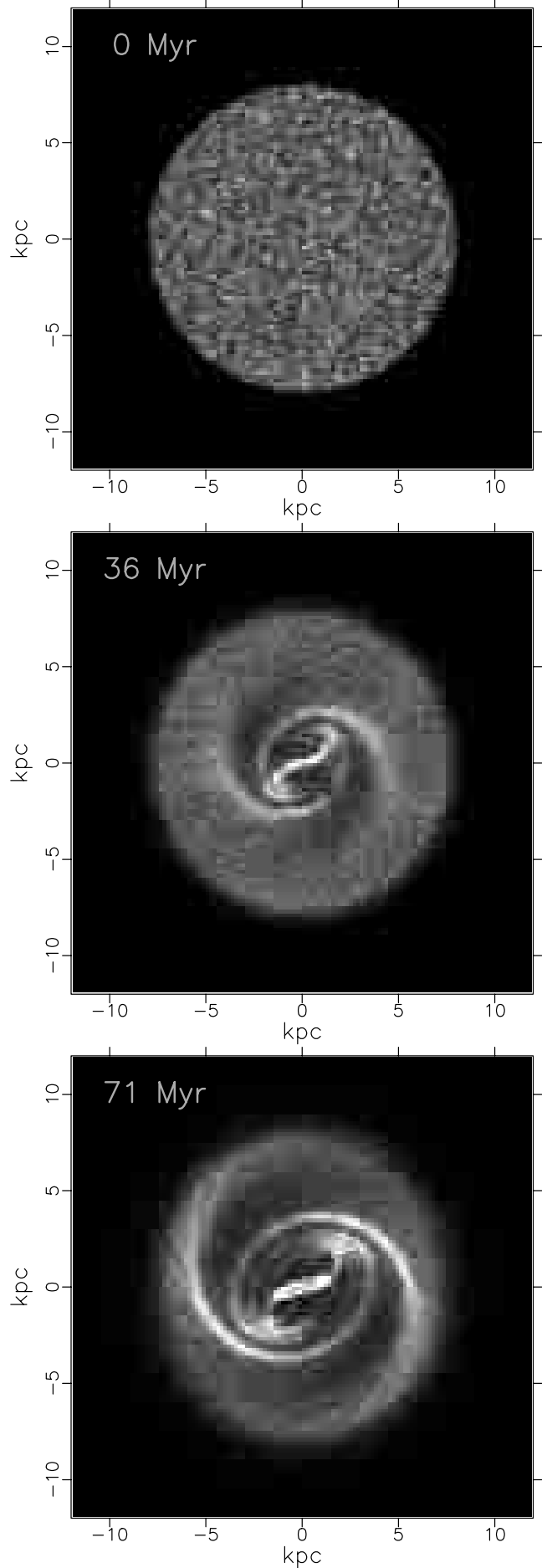
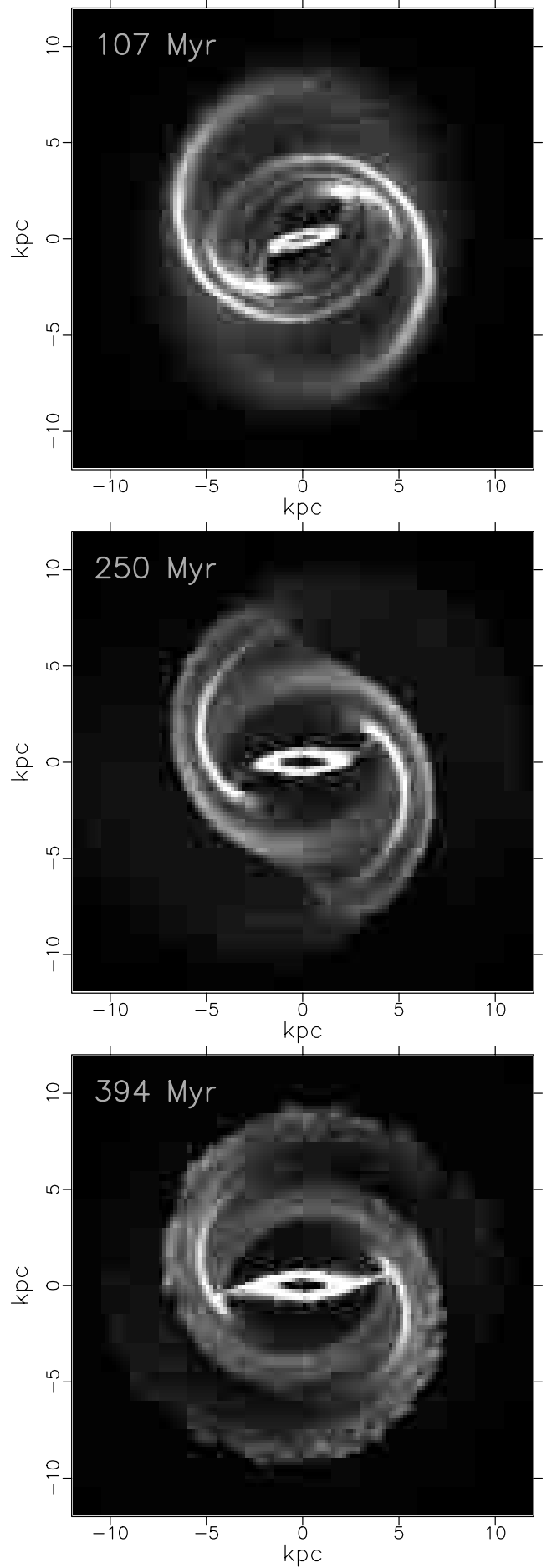

Fig. 3. Gas dynamical evolution in model $\mathrm{E}:\left(\Omega_{\mathrm{b}}, \epsilon_{0}\right)=(0.8,0.10)$. The stellar bar runs horizontally, and the gas rotates counterclockwise. After $t \sim 250 \mathrm{Myr}$ the system reaches a state of quasi-equilibrium.

The ratio of the mass $M_{\mathrm{obs}}$, derived from an observed rotation curve $V_{\text {obs }}$, over the true mass $M_{\text {pot }}$ from the potential is,

$\gamma(R) \equiv \frac{M_{\mathrm{obs}}}{M_{\mathrm{pot}}}\left[=\alpha(R)^{2}\right] ;$

the second equal comes from Eq. (1). Then Eq. (6) becomes equivalent to

$\gamma(R) \geq \gamma_{\text {crit }}$,
A rotation curve rises steeply from a galactic center, having a peak, or at least a shoulder, at an innermost region, then reaching the flat rotation. A central galactic mass is always estimated at the radius of the peak or shoulder in observations (see Sofue et al. 1999). We thus consider the case that the mass is overestimated/underestimated at the radius of the first peak or shoulder. The radius depends on, and changes with a viewing angle for the gas disk (see Fig. 6). Hence, we define a reference

where $\gamma_{\text {crit }}=\alpha_{\text {crit }}^{2}$. 


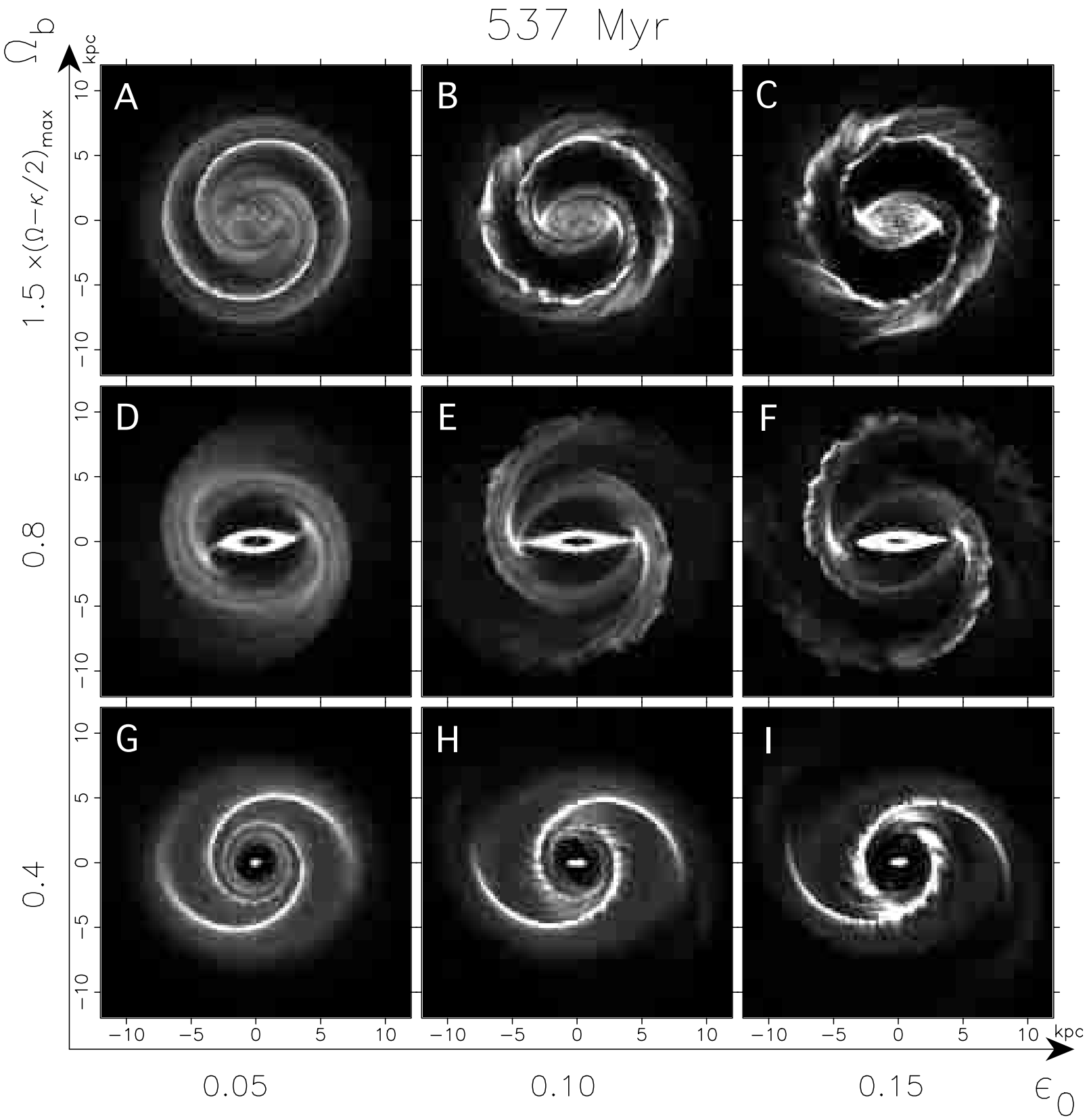

Fig. 4. Final snapshots for all nine models. Different pattern speeds $\Omega_{\mathrm{b}}$ and bar strengths $\epsilon_{0}$ are arranged vertically and horizontally, respectively. The stellar bar runs horizontally, and the gas rotates counterclockwise.

region of $400 \mathrm{pc}<R<2 \mathrm{kpc}^{1}$ to which the above criterion, i.e. Eq. (6), is applied; in our models the first peak or shoulder always fall in this region. If Eq. (6) or (8) is satisfied in this reference region, our observed rotation velocity differs from the true velocity at least by a factor of $\alpha_{\text {crit }}$.

\subsection{Probability of erroneous mass estimation}

We define the probability that the central rotation velocity is larger/smaller by a factor of $\alpha_{\text {crit }}$, as the fraction of viewing angles. We obtain rotation curves by observing the gas

\footnotetext{
1 The absolute values are not important because our results are almost scalable for an arbitrary core radius.
}

disk for viewing angles at ten-degree intervals, and calculate the probabilities for three-hundred snapshots in each simulation. We hereafter describe the probability as $P\left[\alpha>\alpha_{\text {crit }}\right]$ or $P_{\alpha}\left(\alpha_{\text {crit }}\right)$. Figure 7 shows a time evolution of $P_{\alpha}$ in model E. Corresponding to the three evolutionary phases (Sect. 2.4), the probability rises steeply in the linear perturbation phase, approaching a constant value in the transient phase, then reaching the constant in the quasi-steady phase. We also calculate the averaged probability in the quasi-steady phase, i.e. $300-500 \mathrm{Myr}$, and describe it as $\tilde{P}_{\alpha}$. Similarly $P_{\gamma}\left(\gamma_{\text {crit }}\right)$, the probability that the estimated mass differs from the true galactic mass by a factor of $\gamma_{\text {crit }}$, is defined. $\tilde{P}_{\gamma}$ is a time-average of $P_{\gamma}\left(\gamma_{\text {crit }}\right)$.

Figure 8 shows the averaged probability $\tilde{P}_{\alpha}$ as a function of $\alpha_{\text {crit }}$ for all nine models. Different pattern speeds $\Omega_{\mathrm{b}}$ and bar 


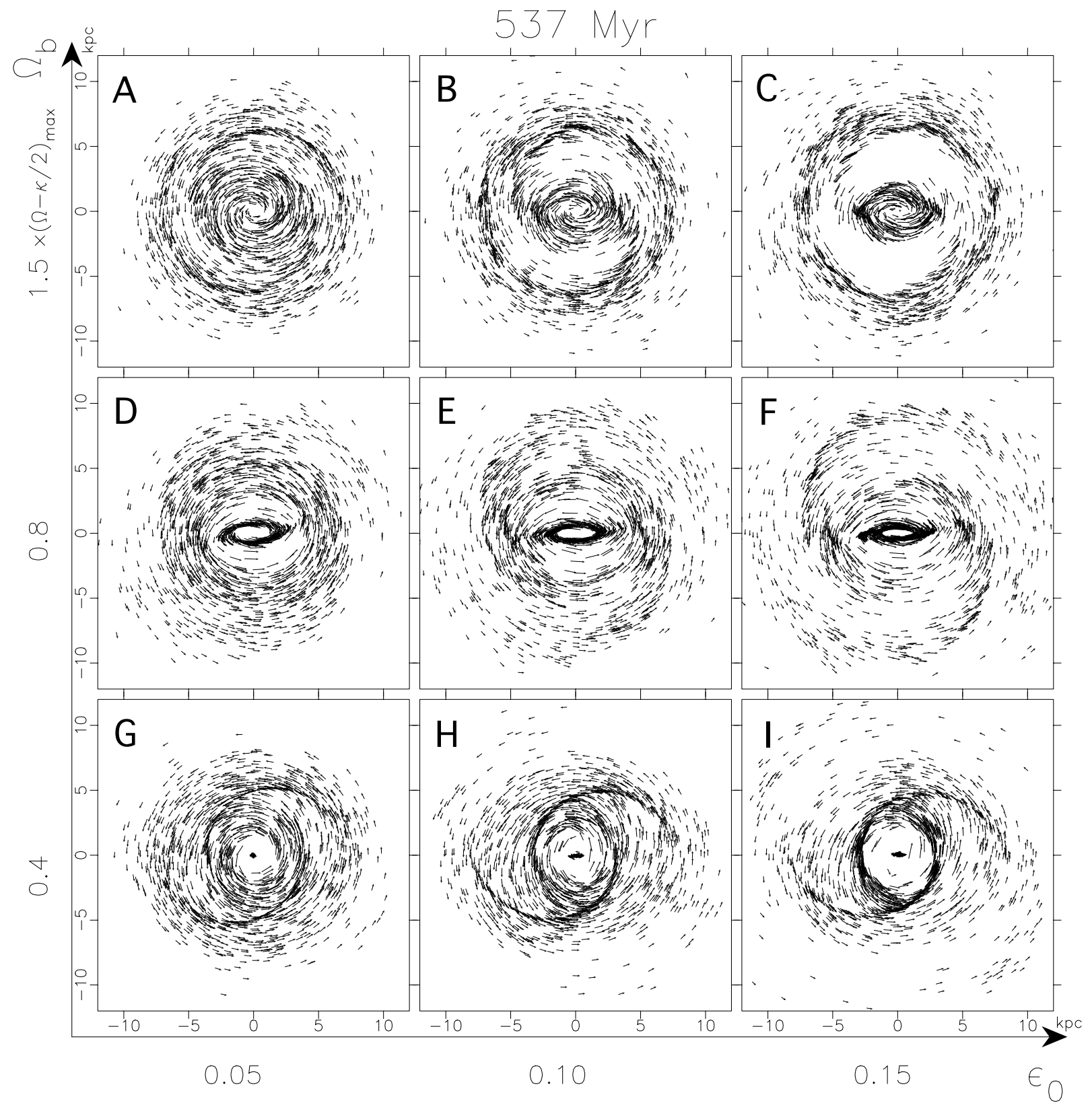

Fig. 5. Final velocity fields for all nine models. The arrangement is the same as in Fig. 3. Arrows are drawn for 1500 out of total 30000 gas particles.

strengths $\epsilon_{0}$ are arranged vertically and horizontally, respectively, as in Fig. 4. If the gas follows pure circular rotation, i.e. $V_{\text {obs }}=V_{\text {pot }}$, then these $\tilde{P}_{\alpha}$-profiles must be a step function: $\tilde{P}_{\alpha}=1$ for $\alpha_{\text {crit }} \leq 1$ and $\tilde{P}_{\alpha}=0$ for $\alpha_{\text {crit }}>1$. However, the non-circular motion changes the $\tilde{P}_{\alpha}$-profiles as seen in the plots. In model E for example, $\tilde{P}_{\alpha}=0.8$ at $\alpha_{\text {crit }}=1.0$ means that $80 \%$ of the rotation curves $V_{\text {obs }}$ observed from random angles would apparently show higher velocities than the true rotation curve $V_{\text {pot }}$ which traces the mass, and another $20 \%$ would show lower velocities than the true one. Thus the overestimation in mass occurs more frequently than the underestimation in model E. $\tilde{P}_{\alpha}=0.0$ at $\alpha_{\text {crit }}=2.4$ indicates that the observed rotation curve cannot be overestimated by more than the factor of 2.4 in model E.
We discussed in Sect. 2.4 that the final structure depends strongly on the pattern speed $\Omega_{b}$ and a little on the bar strength $\epsilon_{0}$. This is also evident in Fig. 8; the global profiles in the same $\Omega_{\mathrm{b}}$ are quite similar, but $\tilde{P}_{\alpha}$ increases slightly with increasing $\epsilon_{0}$. Models A, B and C have no ILRs, not showing nonaxisymmetric structures in the central regions (Sect. 2.4), thus the $\tilde{P}_{\alpha}$-profiles are similar to the step functions. Models D, E and $\mathrm{F}$ show the most prominent streaming motions in their central regions, and therefore they have the largest $\tilde{P}_{\alpha}$. Although models G, H and I have the same $\Omega_{\mathrm{b}}$, the $\tilde{P}_{\alpha}$-profile for model G is different from those for models $\mathrm{H}$ and $\mathrm{I}$, because gaseous $x_{2}$-orbits remain in models $\mathrm{H}$ and $\mathrm{I}$, but not in model $\mathrm{G}$ (see Sect. 2.4). 

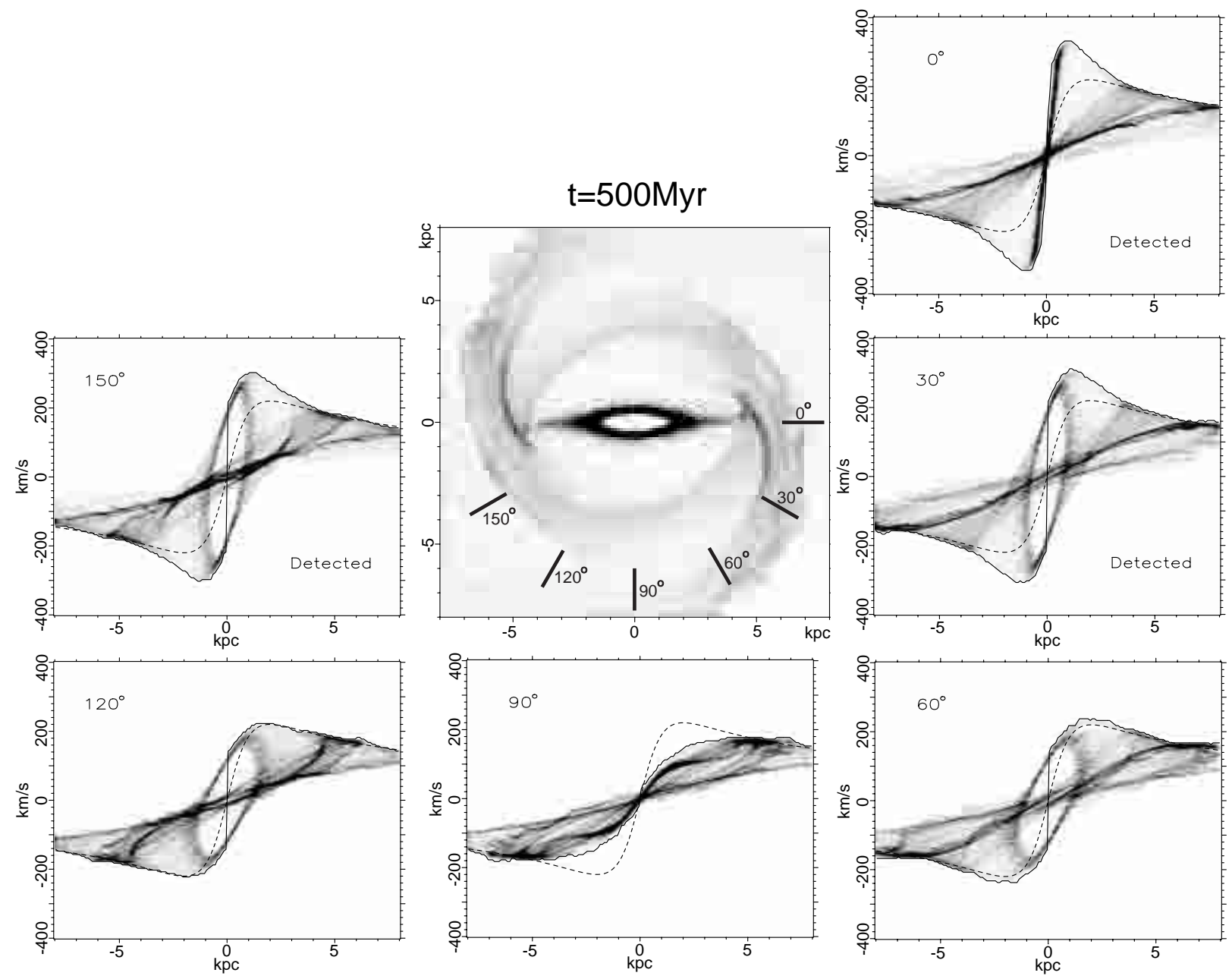

Fig. 6. Position-velocity diagrams and rotation curves in model E, observed from a variety of viewing angles, at $t=500 \mathrm{Myr}$. Solid lines show the distorted rotation curves derived from the calculated p-v diagrams (gray scale), while dashed lines indicate the rotation curve derived from the model potential. The mark "Detected" indicates that our criterion with $\alpha_{\text {crit }}=2$ is satisfied.

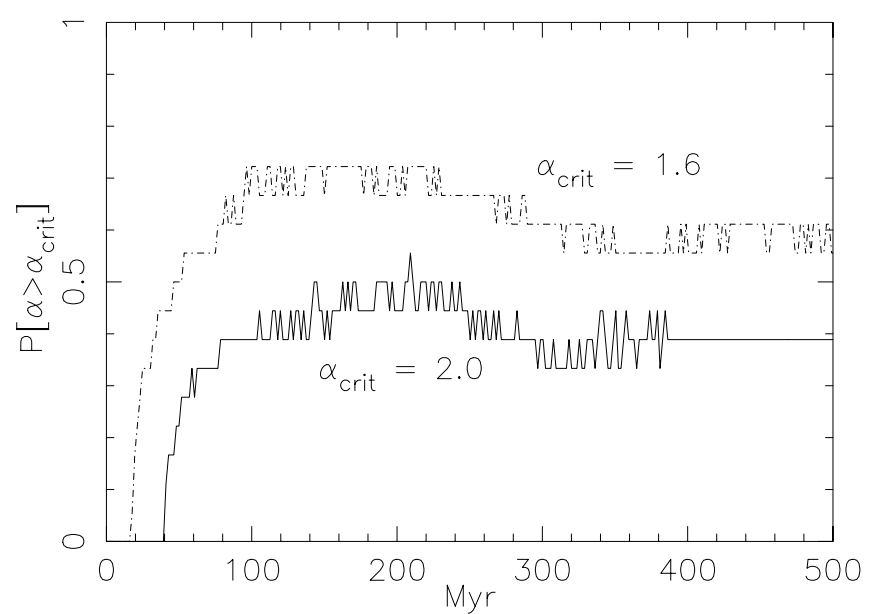

Fig. 7. Change of the probability $P\left[\alpha>\alpha_{\text {crit }}\right]=P_{\alpha}\left(\alpha_{\text {crit }}\right)$ that Eq. (6) will be satisfied. $\alpha_{\text {crit }}=1.6$ and 2.0 indicate the cases that an observed rotation velocity exceeds the true value by a factor of 1.6 and 2.0 times, respectively.

Figure 9 shows the probability $\tilde{P}_{\gamma}$ vs. $\gamma_{\text {crit }}$. All the plots show properties similar to those in Fig. 8. In model E for example, $\tilde{P}_{\gamma}=0.15$ at $\gamma_{\text {crit }}=5.0$ means that the central galactic mass derived from an observed rotation curve is overestimated by a factor of five in the probability of $15 \% . \tilde{P}_{\gamma}$ s in all models become zero at $\gamma_{\text {crit }}=6.0$, meaning that the central mass from an observed rotation curve can be overestimated by at most a factor of six in our models.

\section{Discussion and summary}

Based on gas dynamical calculations in a fixed galactic potential with a weak bar-like distortion, we estimated errors in mass estimation from the rotation curves, and calculated the probability that observations would suffer from such errors. We found that, as well as the final morphologies of gas disks, the probability strongly depends on the pattern speed of a bar $\Omega_{\mathrm{b}}$, and weakly on the bar strength $\epsilon_{0}$. Among our nine models, the probability for the errors becomes maximal for the models with $\Omega_{\mathrm{b}}=0.8 \times(\Omega-\kappa / 2)_{\max }$; the $15 \%$ of them have an error of a factor of five in mass estimation, if we observe the disks from an arbitrary viewing angle, and if we define rotation curves as the highest-velocity envelope of the p-v diagrams. Even in those erroneous cases, the galactic mass is not overestimated by more 


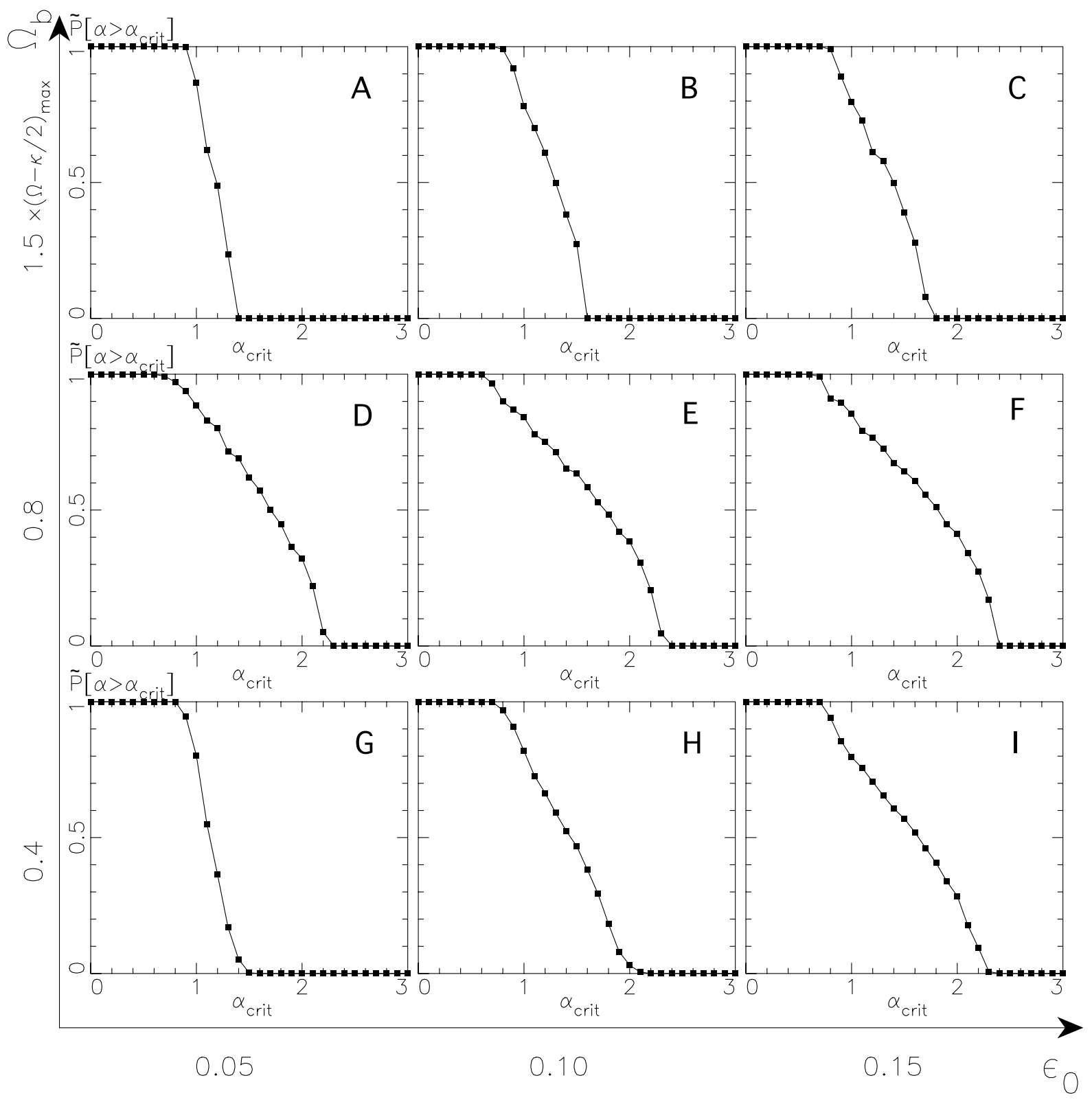

Fig. 8. The probability that an observed rotation velocity would exceed $\alpha_{\text {crit }}$ times that inferred from the galaxy potential. Probability $\tilde{P}_{\alpha}$ is averaged over the time $t=300-500 \mathrm{Myr}$ when the systems are in quasi-steady states. Models with different pattern speeds $\Omega_{\mathrm{b}}$ and bar strengths $\epsilon_{0}$ are arranged vertically and horizontally, respectively.

than a factor of six. In all of our models, the overestimation in mass is more probable than the underestimation. We consider only some particular cases for a weak bar, thus cannot obtain general conclusions. The above estimation however must be a guideline to consider the central galactic mass derived from an observed rotation curve.

Conventionally, rotation curves have been often defined as the peak-intensity velocity or intensity-weighted mean velocity of p-v diagrams. However, Sofue (1996) pointed out that these methods underestimate the rotation velocity, particularly in the central region, because the finite beam size causes the confusion with the gas with lower line-of-sight velocities on the p-v diagram; this effect is also demonstrated in Koda et al. (2002, in their Fig. 15). For rotation curves in highly inclined galaxies, this confusion can not be avoidable. Alternatively, the envelope-velocity of the $\mathrm{p}-\mathrm{v}$ diagram is better suited to trace the central mass distribution (Sofue 1996; Sofue \& Rubin 2001). Therefore we defined the highest-envelope velocity as our rotation curves in the above study. Here we repeat the same analysis for a comparison, using rotation curves derived from the density-weighted mean velocity, and shows the results in Fig. 10. $\tilde{P}_{\gamma}$ s are always less than those in Fig. 9, and are almost zero at $\gamma_{\text {crit }}=1.0$. This means that the mass derived from the mean-velocity rotation curves are almost always underestimated in the central regions of galaxies. These results suggest that the conventional method for deriving rotation curves from p-v diagrams is not also relevant to estimate the mass in galaxies with bar-like distortions. 


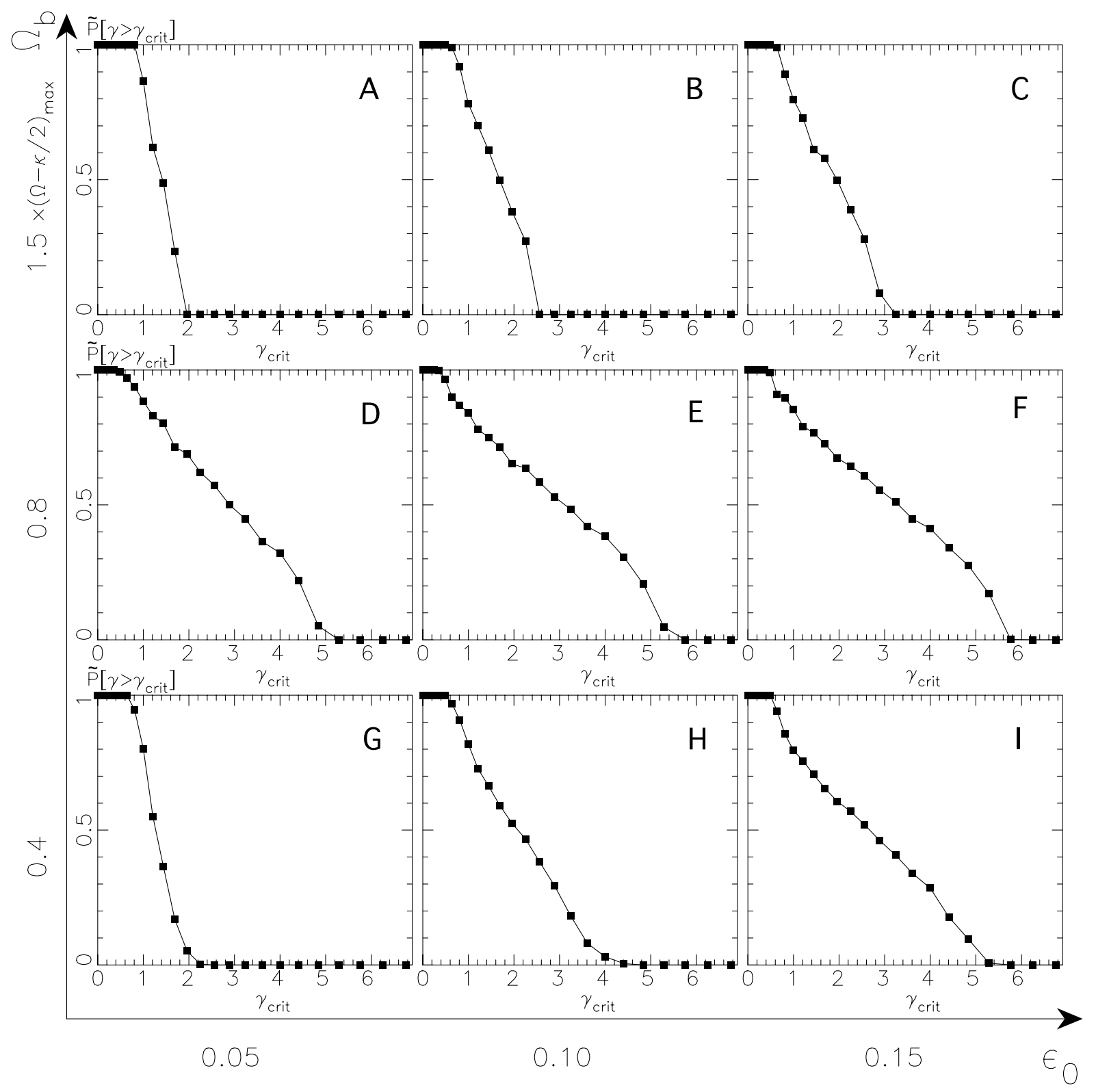

Fig. 9. The probability that an observed mass would exceed $\gamma_{\text {crit }}$ times that inferred from the galaxy potential by chance. Probability $\tilde{P}_{\gamma}$ is averaged in $t=300-500 \mathrm{Myr}$ when the systems are in quasi-steady states. Models with different pattern speeds $\Omega_{\mathrm{b}}$ and bar strengths $\epsilon_{0}$ are arranged vertically and horizontally, respectively.

Sofue et al. (1999) showed that most of the rotation curves rise steeply from the centers, reaching high velocities of about $100-300 \mathrm{~km} \mathrm{~s}^{-1}$ in the innermost regions. Owing to the large fraction of the rotation curves with these high central velocities, they discussed the idea that these velocities should be attributed to massive cores rather than to bars. We may have a chance to statistically clarify whether or not the massive cores exist by comparing a probability such as ours with the observed fraction of rotation curves with high central velocities. When we define the probability $P$ averaged in all types of barred and non-barred galaxies by

$P=\int \tilde{P}_{\alpha}\left(\Omega_{\mathrm{b}}, \epsilon_{0}\right) f\left(\Omega_{\mathrm{b}}, \epsilon_{0}\right) \mathrm{d} \Omega_{\mathrm{b}} \mathrm{d} \epsilon_{0}$ where $f\left(\Omega_{\mathrm{b}}, \epsilon_{0}\right)$ is a distribution function of galaxies with a pattern speed $\Omega_{\mathrm{b}}$ and bar strength $\epsilon_{0}$, the existence of massive cores is confirmed if the fraction of galaxies with the central high velocities is more than $P$. For example, using our maximum calculated probability $\left.\tilde{P}_{\alpha}\right|_{\max }=0.4$ and an observed fraction of barred galaxies $f_{\text {bar }} \sim 0.6$ (Knapen et al. 2000), $P$ could be very roughly calculated as $P<\left.\tilde{P}_{\alpha}\right|_{\max } \times f_{\text {bar }}=0.24$. Of course, we need more intensive studies for a number of barred potentials and parameters, and more precise knowledge of the distribution function of parameters for bars.

Acknowledgements. We are grateful to Y. Sofue for fruitful discussions. We also thank an anonymous referee and H. J. Habing, the editor, for useful comments. J.K. was financially supported by the Japan Society for the Promotion of Science (JSPS) for Young Scientists. 


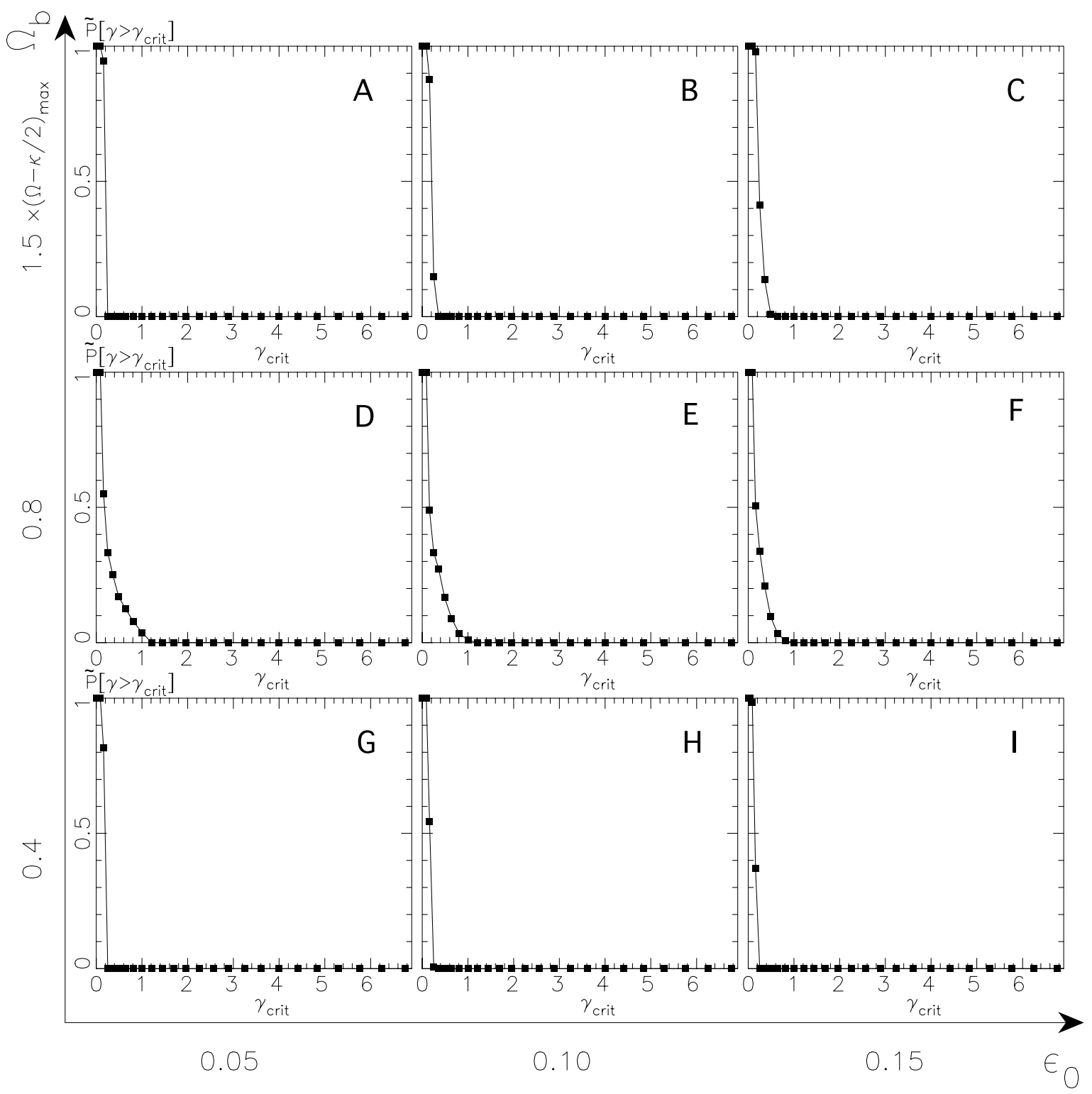

Fig. 10. Same as Fig. 9, but rotation curves are derived by taking the density-weighted mean velocity rather than the most rapidly rotating envelope of the $\mathrm{p}-\mathrm{v}$ diagram.

\section{References}

Athanassoula, E., \& Bureau, M. 1999, ApJ, 522, 699

Balsara, D. S. 1995, J. Comp. Phys., 121, 357

Benz, W. 1990, The Numerical Modeling of Nonlinear Stellar Pulsations (Kluwer Academic Publishers), 269

Bureau, M., \& Athanassoula, E. 1999, ApJ, 522, 686

Fukuda, H., Wada, K., \& Habe, A. 1998, MNRAS, 295, 463

Fukuda, H., Habe, A., \& Wada, K. 2000, ApJ, 529, 109

Gingold, R. A., \& Monaghan, J. J. 1977, MNRAS, 181, 375

Heller, C., \& Shlosman, I. 1994, ApJ, 424, 84

Hernquist, L., \& Katz, N. 1989, ApJS, 70, 419

Hockney, R. W., \& Eastwood, J. W. 1981, Computer Simulation using Particles (McGraw-Hill)

Kent, S. M. 1987, AJ, 93, 816

Knapen, J. H., Shlosman, I., \& Peletier, R. F. 2000, ApJ, 529, 93

Koda, J., Sofue, Y., Kohno, K., et al. 2002, ApJ, 573, 105

Lucy, L. 1977, AJ, 82, 1013

Mathewson, D. S., \& Ford, V. L. 1996, ApJS, 107, 97
Mathewson, D. S., Ford, V. L., \& Buchhorn, M. 1992, ApJS, 81, 413

Monaghan, J. J., \& Lattanzio, J. C. 1985, A\&A, 149, 135

Piner, G. B., Stone, J. M., \& Teuben, P. J. 1995, ApJ, 449, 508

Rubin, V. C., Ford, J. W. K., \& Thonnard, N. 1980, ApJ, 238, 471

Rubin, V. C., Thonnard, N., Ford, W. K. J., et al. 1982, ApJ, 261, 439

Rubin, V. C., Burstein, D., Ford, W. K. J., et al. 1985, ApJ, 289, 81

Sakamoto, K., Okumura, S. K., Ishizuki, S., et al. 1999, ApJS, 124, 403

Sanders, R. H. 1977, ApJ, 217, 916

Sofue, Y. 1996, ApJ, 458, 120

Sofue, Y., Tutui, Y., Honma, M., et al. 1999, ApJ, 523, 136

Sofue, Y., \& Rubin, V. 2001, ARA\&A, 39, 137

Takamiya, T., \& Sofue, Y. 2000, ApJ, 534, 670

Thomas, P. A., \& Couchman, H. M. P. 1992, MNRAS, 257, 11

Wada, K. 1994, PASJ, 46, 165

Wada, K., \& Habe, A. 1992, MNRAS, 258, 82

Wada, K., \& Habe, A. 1995, MNRAS, 277, 433

Wada, K., Sakamoto, K., \& Minezaki, T. 1998, ApJ, 494, 236

Wada, K., Taniguchi, Y., Habe, A., et al. 1994, ApJ, 437, 123 